\title{
Analysis of gene profiles involved in the enhancement of all-trans retinoic acid-induced HL-60 cell differentiation by sesquiterpene lactones identifies asparagine synthetase as a novel target for differentiation-inducing therapy
}

\author{
JU HAN SONG ${ }^{1}$, SEUNG HYUN KIM ${ }^{1}$, KYUNG-MIN CHO ${ }^{1}$, SEUNG YONG HWANG ${ }^{2}$, \\ HYEOUNG-JOON KIM ${ }^{3}$ and TAE SUNG KIM ${ }^{1}$ \\ ${ }^{1}$ Department of Life Sciences, College of Life Sciences and Biotechnology, Korea University, Seoul 136-701; \\ ${ }^{2}$ Division of Molecular and Life Science and GenoCheck Co. Ltd., Hanyang University, \\ Ansan 426-791; ${ }^{3}$ Genome Research Center for Hematopoietic Diseases, \\ Chonnam National University Hwasun Hospital, Hwasun 519-809, Republic of Korea
}

Received October 10, 2013; Accepted November 29, 2013

DOI: 10.3892/ijo.2013.2241

\begin{abstract}
All-trans retinoic acid (ATRA) is one of the most useful drugs in the treatment for acute promyelocytic leukemia (APL), but its adverse effects, which include drug resistance and hypercalcemia are obstacles to achieving complete remission. Our previous study showed that some sesquiterpene lactones (STLs), i.e., helenalin (HE) and parthenolide (PA) but not sclareolide (SC), enhance ATRA-induced differentiation of HL-60 APL cells with no unexpected effects, but the precise mechanism on underlying this synergism is not yet fully understood. In this study, we investigated the distinctive transcriptional profile of cells treated with effective STL compounds, which were identified by comparing the profile with that of cells treated with SC. Genome-wide approaches using cDNA microarrays showed that co-treatment with the differentiation-enhancing STLs HE and PA maximized the transcriptional variation regulated by the suboptimal concentration of ATRA in HL-60 cells. Of the genes of interest, asparagine synthetase was remarkably downregulated by ATRA co-treated with either HE or PA, but not with SC. In an additional analysis for the role of asparagine synthetase, ATRA-mediated HL-60 cell differentiation was enhanced when asparagine in the culture media was depleted by an addition of L-asparaginase, indicating that downregulation of asparagine synthetase gene expression may be involved in the enhanced cell differentiation by STL compounds. These
\end{abstract}

Correspondence to: Dr Tae Sung Kim, Department of Life Sciences, College of Life Sciences and Biotechnology, Korea University, 5-ga, Anam-dong, Seongbuk-gu, Seoul 136-701, Republic of Korea

E-mail: tskim@korea.ac.kr

Key words: All-trans retinoic acid, acute promyelocytic leukemia, asparagine synthetase, differentiation, sesquiterpene lactone results provide useful insight into differentiation-inducing therapy in the treatment of leukemia.

\section{Introduction}

All-trans retinoic acid (ATRA) is a derivative of vitamin A and is most useful in the treatment of acute promyelocytic leukemia (APL) (1-4). This drug has been shown to cause terminal differentiation of immature leukemic blasts by regulating many target genes including retinoic acid receptor, CCAAT/enhancer-binding protein $\beta$ and interferon regulatory factor 1 (5-7). Although it has been established that there is a high rate of complete remission with the administration of ATRA, there are several reports of adverse effects such as differentiation syndrome, hypercalcemia and ATRA resistance (8-11). Therefore, combination therapy of ATRA with alternative medicines has been suggested to minimize these unexpected effects (12).

Sesquiterpene lactone (STL) compounds, which have a lactone ring, are found in a broad range of plants. There is a growing interest in the pharmacological use of STLs. Parthenolide (PA) isolated from Tanacetum parthenium strongly inhibits proinflammatory cytokine-induced signal activation in immune disorders $(13,14)$. Furthermore, the antitumor effects of PA have already been evaluated in vitro and in vivo $(15,16)$. Other STLs such as helenalin (HE) and costunolide also exhibit anti-inflammatory and anticancer activities by greatly inhibiting the transcriptional regulatory activity of nuclear factor $-\kappa \mathrm{B}(\mathrm{NF}-\kappa \mathrm{B})$ and the production of reactive oxygen species (17-20). Although they have a structural similarity, each STL exhibits differential effects in therapeutic applications (21-23). Therefore, molecular dissection of the action mechanism of therapeutically useful STLs is required.

The human leukemia HL-60 cell line has been established as a reasonable model for studying new medicines and their action mechanisms in differentiation-inducing chemotherapy 
(24). The cells are differentiated into monocyte- or granulocytelike cells by stimulating them with 1,25 -dihydroxyvitamin $\mathrm{D}_{3}$ or ATRA, respectively $(1,25)$. Our previous study demonstrated that each STL exhibited different regulatory effects in the enhancement of HL- 60 cell differentiation by combination treatment with ATRA; i.e., PA and HE synergized the ATRAinduced HL-60 cell differentiation into a granulocytic lineage but SC did not (26).

In this study, we attempted to identify the molecular events that occurred when the granulocytic differentiation of HL-60 cells was enhanced by the addition of the STLs, such as PA, HE and SC. To address the question, we used a cDNA microarray-based genome-wide approach and compared data sets obtained from microarray analyses for differentiationinducing and non-inducing agents.

\section{Materials and methods}

Cell line and reagents. Human HL-60 cells were obtained from American Type Culture Collection (Rockville, MD, USA) and cultured in RPMI-1640 medium supplemented with heat-inactivated $10 \%$ fetal bovine serum (Omega Scientific, Tarzana, CA, USA) and antibiotics at $37^{\circ} \mathrm{C}$ in a humidified $5 \% \mathrm{CO}_{2}$ incubator. To maintain exponential growth, cells were seeded at a concentration of $1 \times 10^{5}$ cells $/ \mathrm{ml}$ and sub-cultured every 3-4 days. ATRA, PA, SC and phorbol 12-myristate 13-acetate were from Sigma (St. Louis, MO, USA). HE and L-asparaginase (L-ASNase) were purchased from Enzo Life Sciences (Farmingdale, NY, USA) and Aviva Systems Biology (San Diego, CA, USA), respectively.

Nitroblue tetrazolium (NBT) reduction assay and morphological study. HL-60 cells at a concentration of $1.5 \times 10^{5} /$ $\mathrm{ml}$ were cultured for $72 \mathrm{~h}$ in the presence of ATRA and/or sesquiterpene lactones and L-ASNase. At the end of treatment, the cells were harvested by a centrifugation and incubated in PBS buffer containing 0.1\% NBT (USB, Cleveland, OH, USA) and $200 \mathrm{ng} / \mathrm{ml}$ PMA for $1 \mathrm{~h}$ to allow the cells to form a blueblack nitroblue formazan. The differentiation-positive cells were accessed under a light microscope. At least 200 cells were counted for each culture sample, and the results were expressed as a relative percentage of NBT-positive cells to total cells.

Flow cytometric measurement. At the end of culture, cells were collected, washed with ice-cold PBS buffer and labeled with PE-conjugated CD11b monoclonal antibody (BD Bioscience, San Jose, CA, USA) at room temperature for $15 \mathrm{~min}$. Fluorescent intensity was analyzed by flow cytometric measurement using BD FACSCalibur.

cDNA microarray analysis. HL-60 cells were treated with $50 \mathrm{nM}$ ATRA alone or combination with HE, PA, or SC for $24 \mathrm{~h}$. Total RNA from the cultures were isolated using TRIzol reagent (MRC, Cincinnati, OH, USA). For DNA microarray assay, fluorescence-labeled cDNA probes were obtained from $30 \mu \mathrm{g}$ of total RNA by using SuperScript II reverse transcriptase (Gibco BRL) in a total reaction volume of $30 \mu \mathrm{l}$ and applied to human $8.5 \mathrm{~K}$ cDNA microarrays. The sample from untreated HL-60 cells was used as a reference for each chip assay. The experimental and analytical procedures were done as previously described (27).

$R N A$ preparation and reverse transcription-polymerase chain reaction ( $R T-P C R)$. The cDNA was obtained from 1 to $1.5 \mu \mathrm{g}$ of total RNA by the RocketScript RT kit (Bioneer, Daejeon, Korea). The RT product ( $1 \mu \mathrm{l})$ was applied to each PCR reaction with the following primer sets: asparagine synthetase (ASNS; forward, 5'-acagaaggattggctgcctt-3'; reverse, 5'-cctctcactctcctcctcgg-3'), activating transcription factor 4 (ATF4); forward, 5'-aacagcaaggaggatgcctt-3'; reverse, 5'-gtgctgaggagaccccagat-3'), ATF5 (forward, 5'-ttggatactctggacttgct-3'; reverse, 5'-tccttgacgtactggatctc-3') and $\beta$-actin (forward, 5'-agcgggaaatcgtgcgtg-3'; reverse, 5 '-cagggtacatggtggtgcc-3'). The final products were analyzed on a $1.2 \%$ agarose gel with ethidium bromide staining.

Statistical analysis. The results were obtained from at least three-independent experiments. Statistical significance of the data was determined using a paired Student's t-test. A P-value $<0.05$ was considered statistically significant.

\section{Results}

Differential enhancing effects of STLs on ATRA-induced HL-60 cell differentiation. To confirm the effects of STL compounds on ATRA-induced leukemia cell differentiation, HL-60 cells were treated with one of three different STLs, helenalin (HE), parthenolide (PA) or sclareolide (SC), with or without a suboptimal concentration dose $(50 \mathrm{nM})$ of ATRA. As shown in Fig. 1A, HE itself induced the differentiation of HL-60 cells. Both HE and PA strongly enhanced the effect of ATRA in inducing the differentiation, but SC did not. Similarly, the surface expression of CD11b, a marker antigen of general myeloid differentiation, was increased in the cells by combination treatment of ATRA with either HE or PA (Fig. 1B).

Profiles of genes involved in the enhancement of ATRAinduced differentiation by STLs. To investigate the mechanism by which ATRA-induced HL-60 cell differentiation was enhanced by combination treatment with HE or PA, we used cDNA microarray analyses of cells treated with $50 \mathrm{nM}$ ATRA alone or during co-treatment with HE, PA or SC. Microarray analyses showed that treatment with ATRA alone resulted in transcriptional changes of 111 genes, compared with the basal levels in unstimulated cells. As expected, co-treatment with ATRA and STLs resulted in transcriptional changes in greater number of genes, allowing alterations in an increased number of genes including the gene observed in ATRA-treated HL-60 cells (Fig. 2A). Furthermore, the number of genes influenced by the STLs ( 152 by PA, 257 by HE and 140 by SC) seems to reflect the degree by which the STL enhanced differentiation, implying that the enhancing potential of an STL in ATRA-induced HL-60 cell differentiation depends on the participation of these extra genes.

To further investigate how HE and PA, but not SC, enhance ATRA-induced granulocytic differentiation of HL-60 cells, we sought a common set of genes that were influenced in both the cells treated with HE and with PA, but not in cells treated 

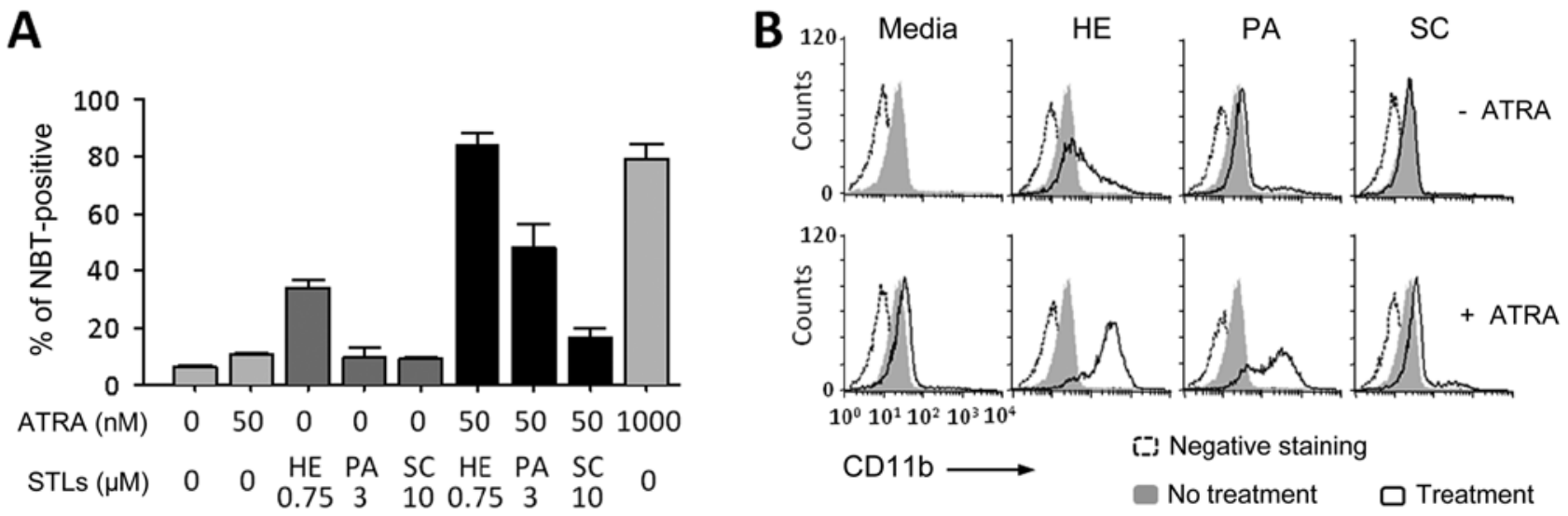

Figure 1. Differential enhancing effects of STLs in ATRA-induced HL-60 cell differentiation. HL-60 cells were incubated in the presence of the drugs indicated, for $72 \mathrm{~h}$. (A) At the end of culture, an NBT assay was performed to determine the differentiation rate. The cells stimulated with $1 \mu \mathrm{M}$ ATRA are shown as an experimental control. Data are expressed as the mean $\pm \mathrm{SD}$ from three individual experiments. (B) At the same time, each cell sample was stained with PE-conjugated anti-CD11b antibody and analyzed using flow cytometric measurement. Representative data of three different experiments with similar results are shown.
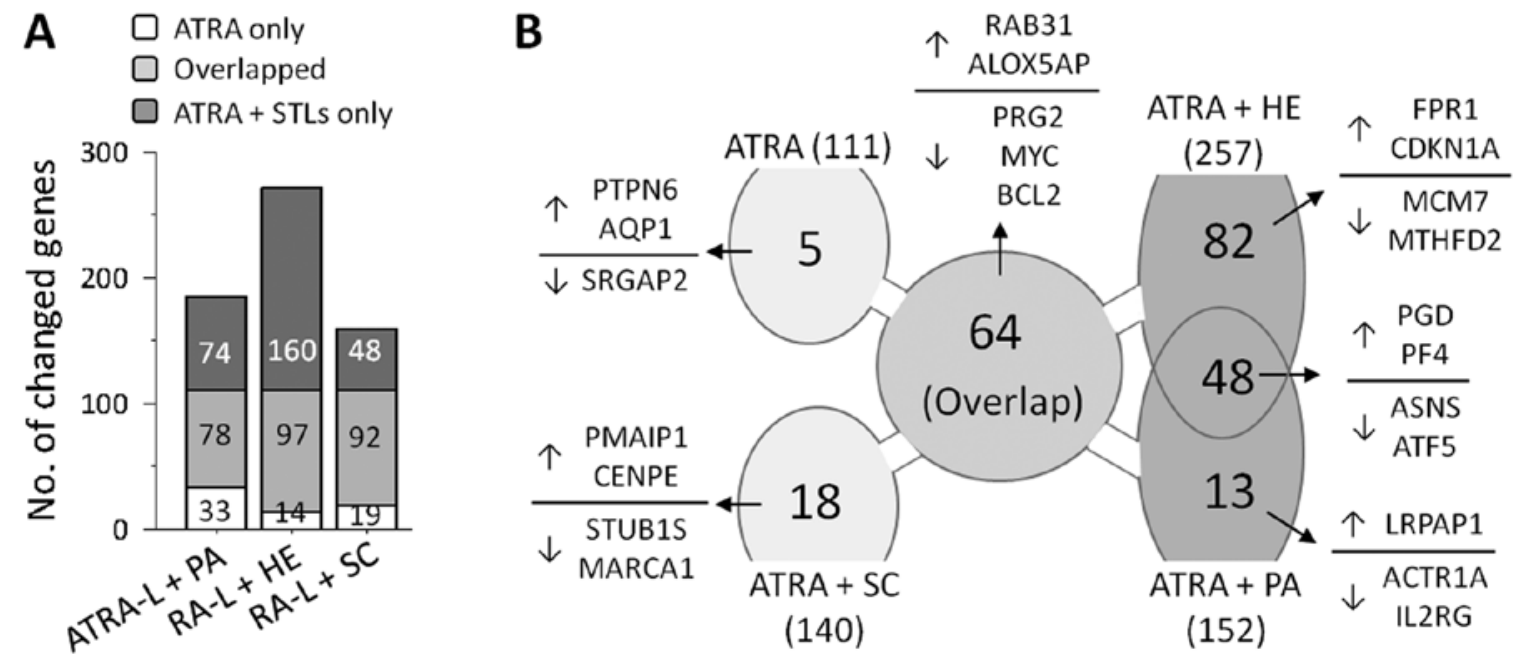

Figure 2. Distribution of genes distinctly regulated by treating with STLs in the presence of ATRA. Gene expression profiles were obtained by cDNA microarray analysis of total RNA isolated from the HL-60 cells, which were stimulated with the indicated drugs for $24 \mathrm{~h}$. Primary data were processed and normalized to data from the non-stimulated control group. Genes that were expressed at the mRNA level at greater than twice or less than half that of the control were considered meaningful. (A) The genes selected from each culture condition were compared with those from the cells treated with ATRA alone. The numbers of relevant genes are indicated. (B) Gene distributions were obtained by comparing the gene content from each culture. The abbreviated names on the diagram are representative genes picked from the indicated groups.

with ATRA alone or co-treated cells with ATRA and SC. As shown in Fig. 2B, 48 genes satisfied the criteria and they are summarized in Table I. Twenty-four of these genes, including phosphogluconate dehydrogenase $(P G D)$ and platelet factor 4 (PF4), were upregulated, whereas the remaining 20, including $A S N S$ and ATF5, were downregulated.

Downregulation of ASNS in the enhancement of ATRAinduced differentiation by STLs. Based on the genome-wide profiles from cDNA chip analysis, we chose ASNS as a target gene for further study in relation to the differentiation of HL-60 cells. We firstly validated the STL-induced change in ASNS mRNA expression using RT-PCR. As shown in Fig. 3, ASNS expression was downregulated in the cells treated with HE or PA alone, as well as in the cells co-treated with ATRA, whereas no difference was found between cells treated with
SC and untreated cells. We also determined the levels of two transcription factors, ATF4 and ATF5, which are known to regulate transcription of $A S N S(28,29)$. In our microarray analysis, like $A S N S$, both genes were also suppressed by HE and PA when combined with ATRA (Table I). This finding suggests that the ASNS may play a role in the differentiation of HL-60 cells.

Enhancement of HL-60 cell differentiation by depletion of $L$-asparagine. It is well known that the protein encoded by ASNS plays an important role in asparagine synthesis. Therefore, to investigate the effect of lower ASNS expression on ATRA-induced cell differentiation, we attempted to create a similar condition by using L-asparaginase (L-ASNase). Treatment with either a suboptimal dose of ATRA $(50 \mathrm{nM})$ or L-ASNase $(0.1 \mathrm{U} / \mathrm{ml})$ had little effect on the expression of 
Table I. Up- or downregulated genes by co-treatment of RA with either PA or HE, but not with SC.

Fold-changes (v.s. no treatment)

GenBank no.

Gene name (40 of 48 genes)

RA-L RA-L + PA RA-L + HE RA-L + SC

Downregulated

AA894927

AA237029

AA419177

AU147203

AI346878

AA213793

AA496253

AW001765

AA447748

AW057866

AA676458

AI951501

AA683050

AW075605

AA167113

AA424912

AI005610

AI369144

AA504475

AA600217

Upregulated

AA598759

M81750

AI954012

AA454104

T97181

AA775264

AI360772

H89664

AA973730

AF020056

AA448157

M80427

AA451863

AA453789

AI000188

T57791

U62795

AA453471

R44739

AA486532
Asparagine synthetase

Homo sapiens cDNA FLJ33469 fis, clone BRAMY2002005

Solute carrier family 7 , member 5

C21orf19-like protein

Sodium channel, non-voltage-gated $1, \beta$ (Liddle syndrome)

KIAA0336 gene product

Activating transcription factor 5

Ribosomal protein L23a

Dihydrolipoamide dehydrogenase

Eukaryotic translation initiation factor 3 , subunit $7 \xi$

Lysyl oxidase-like 2

Ribosomal protein L12

Ribosomal protein S8

Ribosomal protein L9

Homo sapiens cDNA FLJ11689 fis, clone HEMBA1004977

Karyopherin (importin) $\beta 1$

Ribosomal protein L13a

Eukaryotic translation initiation factor $4 \mathrm{E}$ binding protein 1

Mitochondrial ribosomal protein L32

Activating transcription factor 4

Phosphogluconate dehydrogenase

Myeloid cell nuclear differentiation antigen

Adenylyl cyclase-associated protein

Charot-Leyden crystal protein

Platelet factor 4

Echinoderm microtubule associated protein like 2

Myosin IF

Amyloid $\beta$ (A4) precursor-like protein 2

Death-associated protein kinase 3

WD repeat domain 1

Cytochrome P450, subfamily I, polypeptide 1

Androgen-regulated protein FAR-17 (hamster)

CD4 antigen (p55)

Homo sapiens cDNA FLJ36109 fis, clone TESTI2021911

UDP glycosyltransferase 2 family, polypeptide B7

Toll-like receptor 2

Ubiquitin ligase Pub1(yeast)/NEDD-4 isolog(human)

GM2 ganglioside activator protein

Grancalcin, EF-hand calcium binding protein

Major histocompatibility complex, class II, DP $\beta 1$
0.72

0.54

0.69

0.64

0.55

0.58

0.65

0.69

0.53

0.62

0.51

0.73

0.89

0.77

0.82

0.77

0.77

0.78

0.72

0.81

1.79

1.77

1.98

1.58

1.67

1.86

1.89

1.46

1.80

1.83

1.48

1.78

1.91

1.77

1.77

1.81

1.67

1.96

1.49

1.55
0.29

0.22

0.36

0.27

0.30

0.25

0.37

0.38

0.34

0.40

0.47

0.41

0.43

0.46

0.49

0.43

0.44

0.49

0.48

0.48

2.89

2.18

2.45

2.13

2.57

2.17

2.47

2.21

2.07

2.18

2.16

2.11

2.10

2.13

2.17

2.20

2.05

2.35

2.23

2.13
0.13

0.24

0.18

0.29

0.29

0.34

0.32

0.38

0.42

0.37

0.30

0.37

0.40

0.38

0.34

0.42

0.43

0.39

0.40

0.42

4.07

3.15

2.87

3.14

2.62

2.90

2.55

2.75

2.79

2.66

2.66

2.71

2.72

2.61

2.54

2.47

2.62

2.31

2.35

2.36
0.76

0.53

0.62

0.61

0.56

0.72

0.57

0.69

0.62

0.50

0.54

0.56

0.60

0.60

0.57

0.67

0.62

0.72

0.65

0.81

1.93

1.57

1.93

1.35

1.60

1.59

1.76

1.85

1.80

1.77

1.67

1.78

1.91

1.80

1.71

1.68

1.78

1.78

1.61

1.27
CD11b on the cell surface, while a combination of both the drugs increased the expression of this antigen (Fig. 4A). The combined effect of ATRA and L-ASNase was confirmed by a NBT reduction assay (Fig. 4B). On examining nuclear 


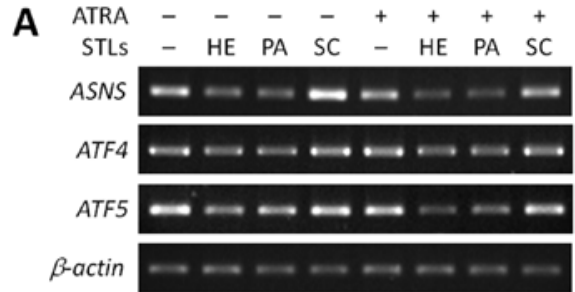

B

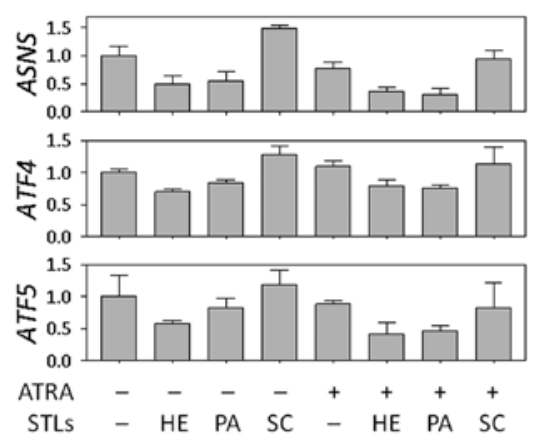

Figure 3. Effect of sesquiterpene lactones on asparagine synthetase gene expression in HL-60 cell differentiation. Total RNA was isolated from the HL-60 cells, which were stimulated for $24 \mathrm{~h}$ with the drugs indicated, and was reverse transcribed into cDNA. PCR-amplified products from the cDNA template were separated on $1.2 \%$ agarose gels and visualized under UV light $\beta$-actin was used as an internal loading control. (A) A representative of three independent experiments is shown. (B) The relative expression levels of each gene were determined by comparing them with that of $\beta$-actin and were expressed as the mean \pm SD. morphology by Giemsa staining, we also observed a slight increase in the cytoplasm to nucleus ratio and that the nuclei were multilobed after treatment with ATRA and L-ASNase (Fig. 4C). Additionally, we examined whether depletion of asparagine could enhance differentiation in HL-60 cells treated with both ATRA and HE. To address this aim, HL-60 cells were treated with ATRA and a lower dose of $\mathrm{HE}(0.3 \mu \mathrm{M})$ in the presence or absence of L-ASNase. As shown in Fig. 4D and $\mathrm{E}$, the addition of L-ASNase to the combination of ATRA and HE strongly increased the number of NBT-positive cells and the levels of the CD11b expression on the cell surface.

\section{Discussion}

Considerable research has been performed on the use of natural STLs as treatments for diverse conditions, including inflammation and cancer. Similar to the results reported for the therapeutic uses of STLs for these diseases, STLs are also effective in differentiation-inducing chemotherapy for leukemia, via NF- $\kappa$ B inhibition $(26,30)$. However, the molecular mechanisms underlying the differentiation-enhancing effects of STLs have not yet been fully elucidated. In this study, our DNA microarray-based approach identified transcriptional reprogramming in APL cells with STL-enhanced granulocytic differentiation. Furthermore, the concurrent application of this technology also identified gene factors that
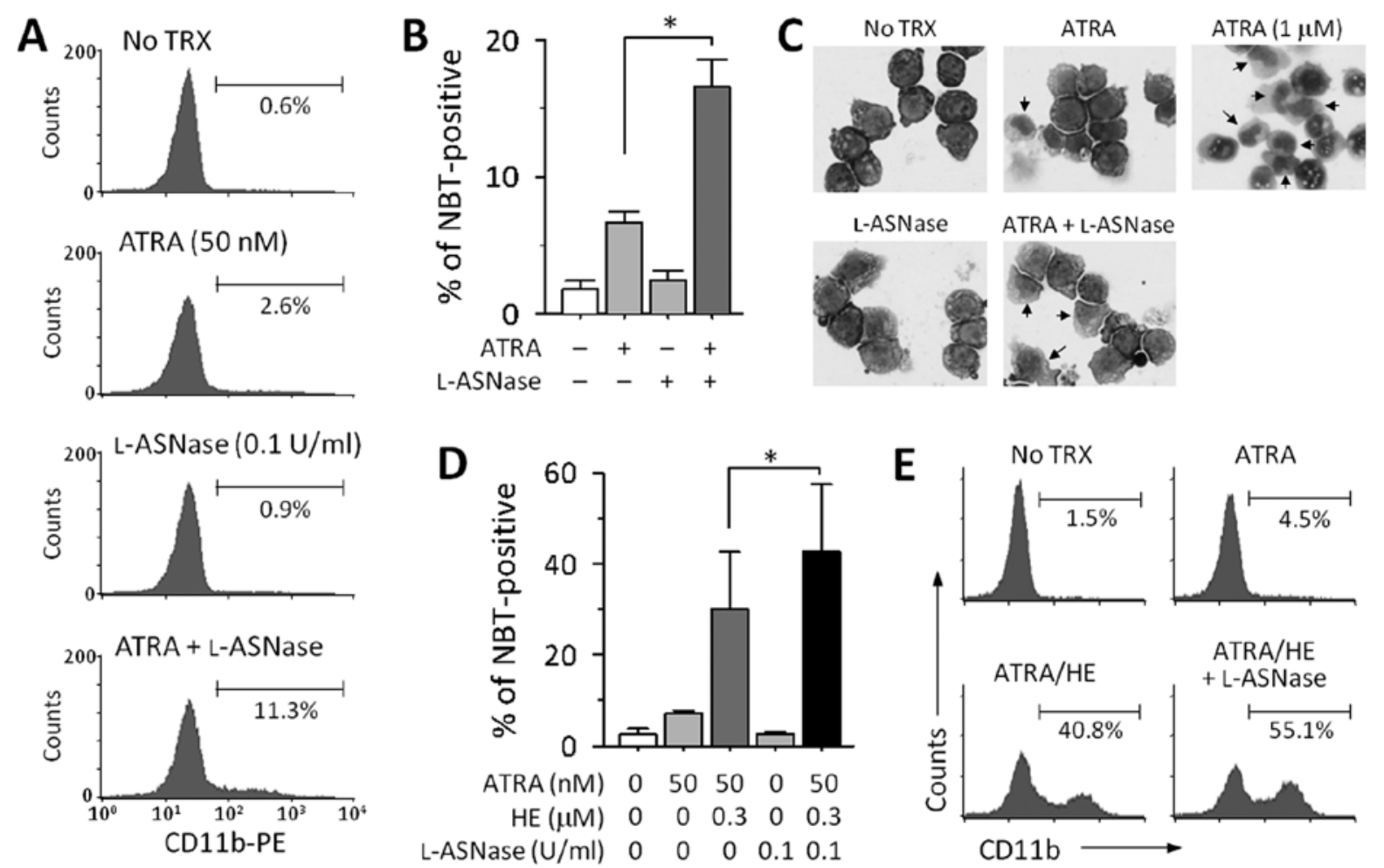

Figure 4. Effect of depletion of asparagine in ATRA-induced HL60 cell differentiation. (A-C) HL-60 cells were treated with 0.1 U/ml L-ASNase in the presence or absence of $50 \mathrm{nM}$ ATRA. At the end of culture for $72 \mathrm{~h}$, the cells were examined with several assays to determine the extent of granulocytic differentiation. (A) CD11b expression on the cell surface was evaluated by flow cytometric analysis. Three different representative experiments are shown. (B) The result from the NBT reduction assay is expressed as the mean \pm SD from three independent experiments ("P<0.01). (C) After cell staining with Giemsa solution, changes in nuclear morphology were observed under a light microscope (x160). Arrows indicate the cells undergoing granulocytic differentiation was polymorphonuclear. (D) The enhancing effect of 1-ASNase on HL-60 cell differentiation by combination treatment with ATRA and HE was determined by an NBT assay. The result is expressed as the mean \pm SD value from four independent experiments $\left({ }^{*} \mathrm{P}<0.05\right)$. The cells incubated in the presence of high doses $(1 \mu \mathrm{M})$ of ATRA are shown as a positive control. (E) From the same cell samples, CD11b expression was determined by flow cytometric measurement. Representative data from three different experiments are shown. 
can lead to differential sensitizing effects between the active STLs (i.e., PA and HE) and the non-active STL (i.e., SC) on ATRA-induced HL-60 cell differentiation.

Using DNA microarrays (although the gene content on the chip did not fully cover all human genes), we identified hundreds of genes that exhibit $>2$-fold changes in the level of transcription in the cells stimulated with a suboptimal concentration of ATRA alone or a combination of ATRA with an STL, compared with non-stimulated reference cells. Interestingly, the number of affected genes was proportional to the degree of enhancement of differentiation (Fig. 2). The gene populations selected from the cells treated with either ATRA alone or in combination with SC overlapped with each other. Furthermore, a large proportion of the genes were subordinate to the gene subsets that were picked from the cells treated with effective STLs, especially HE. These observations agree with our hypothesis that $\mathrm{HE}$ and PA, but not SC, effectively enhance ATRA-induced differentiation by maximizing the degree of transcriptional changes as well as by increasing the number of genes that are involved in HL-60 cell maturation.

In the transcription profiles associated with the enhanced differentiation of HL-60 cells by PA and HE, the outstanding alteration was the down-modulation of $A S N S$, accompanied by the decreased level of ATF4 and ATF5, which are known to be positive transcriptional regulators of ASNS $(28,29)$. ASNS, which encodes asparagine synthetase, has been reported to be aberrantly expressed in many kinds of cancers, including acute lymphoid leukemia (ALL). Since asparagine, which is synthesized by this protein, allows cancer cells to grow rapidly, there is growing interest in targeting asparagine synthetase as a cancer cure $(31,32)$. Indeed, depletion of this amino acid by treatment with L-ASNase is currently used for patients with $\operatorname{ALL}(33,34)$. In this study, we manipulated the levels of L-ASNase without manipulating the gene (ASNS), to investigate the role of the enzyme in HL-60 cell differentiation. The result indicated that depletion of the end product of ASNS, that is, asparagine, in the culture medium was sufficient to enhance ATRA-induced HL-60 cell differentiation. The report by Hongo et al showed that asparagine synthetase activity was decreased when leukemia cells were stimulated with compounds that induce differentiation, implying that asparagine has a potential effect on culture conditions (35). Another study also reported an analogous observation that a decrease in ASNS expression was paralleled by the extent of maturation of HL-60 cells that was induced by 12-O-tetradecanoylphorbol-13-acetate (TPA) (36). The researchers additionally demonstrated the synergistic induction of apoptotic cell death by adding L-ASNase in the presence of TPA, but did not mention a role of L-ASNase in cell differentiation. Although the details of how L-ASNase enhances ATRA-induced leukemia cell differentiation need to be further studied, to our knowledge, our findings provide the first evidence that L-ASNase can enhance differentiation induced in leukemia cells. In addition, the ability of some STLs to downregulate ASNS transcription may offer a therapeutic strategy for L-ASNase-resistant acute leukemia.

Taken together, our use of high-throughput microarray analysis demonstrates the existence of sets of genes that are differentially involved in the enhancement of ATRA-induced APL differentiation by effective STLs; this information also suggests a therapeutic use of STLs. Our additional observa- tion that depletion of asparagine by L-ASNase synergistically enhanced HL-60 cell differentiation by ATRA may also be a valuable strategy in the treatment of leukemia, especially APL.

\section{Acknowledgements}

This study was supported, by the grant of the National Project for Personalized Genomic Medicine, Ministry for Health and Welfare (no. A111218-GM06), the National Research Foundation of Korea (NRF) grant funded by the Korea government (MSIP) (no. 2005-0049410), and a Korea University grant.

\section{References}

1. Breitman TR, Selonick SE and Collins SJ: Induction of differentiation of the human promyelocytic leukemia cell line (HL-60) by retinoic acid. Proc Natl Acad Sci USA 77: 2936-2940, 1980.

2. Breitman TR, Collins SJ and Keene BR: Terminal differentiation of human promyelocytic leukemic cells in primary culture in response to retinoic acid. Blood 57: 1000-1004, 1981.

3. Flynn PJ, Miller WJ, Weisdorf DJ, Arthur DC, Brunning R and Branda RF: Retinoic acid treatment of acute promyelocytic leukemia: in vitro and in vivo observations. Blood 62: 1211-1217, 1983.

4. Huang ME, Ye YC, Chen SR, et al: Use of all-trans retinoic acid in the treatment of acute promyelocytic leukemia. Blood 72: $567-572,1988$.

5. Collins SJ, Robertson KA and Mueller L: Retinoic acid-induced granulocytic differentiation of HL-60 myeloid leukemia cells is mediated directly through the retinoic acid receptor (RAR-alpha). Mol Cell Biol 10: 2154-2163, 1990.

6. Duprez E, Wagner K, Koch H and Tenen DG: C/EBPbeta: a major PML-RARA-responsive gene in retinoic acid-induced differentiation of APL cells. EMBO J 22: 5806-5816, 2003.

7. Matikainen S, Ronni T, Hurme M, Pine R and Julkunen I: Retinoic acid activates interferon regulatory factor-1 gene expression in myeloid cells. Blood 88: 114-123, 1996.

8. Bennett MT, Sirrs S, Yeung JK and Smith CA: Hypercalcemia due to all trans retinoic acid in the treatment of acute promyelocytic leukemia potentiated by voriconazole. Leuk Lymphoma 46: 1829-1831, 2005.

9. Imaizumi M, Suzuki H, Yoshinari M, et al: Mutations in the E-domain of RAR portion of the PML/RAR chimeric gene may confer clinical resistance to all-trans retinoic acid in acute promyelocytic leukemia. Blood 92: 374-382, 1998.

10. Montesinos P, Bergua JM, Vellenga E, et al: Differentiation syndrome in patients with acute promyelocytic leukemia treated with all-trans retinoic acid and anthracycline chemotherapy: characteristics, outcome, and prognostic factors. Blood 113: 775-783, 2009.

11. Shao W, Benedetti L, Lamph WW, Nervi C and Miller WH Jr: A retinoid-resistant acute promyelocytic leukemia subclone expresses a dominant negative PML-RAR alpha mutation. Blood 89: 4282-4289, 1997

12. Lengfelder E, Saussele S, Weisser A, BüchnerT and Hehlmann R: Treatment concepts of acute promyelocytic leukemia. Crit Rev Oncol Hematol 56: 261-274, 2005.

13. Hehner SP, Hofmann TG, Dröge W and Schmitz ML: The antiinflammatory sesquiterpene lactone parthenolide inhibits NF-kappa B by targeting the I kappa B kinase complex. J Immunol 163: 5617-5623, 1999.

14. Sobota R, Szwed M, Kasza A, Bugno M and Kordula T: Parthenolide inhibits activation of signal transducers and activators of transcription (STATs) induced by cytokines of the IL-6 family. Biochem Biophys Res Commun 267: 329-333, 2000.

15. Oka D, Nishimura K, Shiba M, et al: Sesquiterpene lactone parthenolide suppresses tumor growth in a xenograft model of renal cell carcinoma by inhibiting the activation of NF-kappaB. Int J Cancer 120: 2576-2581, 2007.

16. Sohma I, Fujiwara Y, Sugita Y, et al: Parthenolide, an NF-кB inhibitor, suppresses tumor growth and enhances response to chemotherapy in gastric cancer. Cancer Genomics Proteomics 8: 39-47, 2011. 
17. Hoffmann R, von Schwarzenberg K, López-Antón N, Rudy A Wanner G, Dirsch VM and Vollmar AM: Helenalin bypasses Bcl-2-mediated cell death resistance by inhibiting NF- $\kappa \mathrm{B}$ and promoting reactive oxygen species generation. Biochem Pharmacol 82: 453-463, 2011.

18. Kassuya CA, Cremoneze A, Barros LF, et al: Antipyretic and anti-inflammatory properties of the ethanolic extract, dichloromethane fraction and costunolide from Magnolia ovata (Magnoliaceae). J Ethnopharmacol 124: 369-376, 2009.

19. Lyss G, Knorre A, Schmidt TJ, Pahl HL and Merfort I: The anti-inflammatory sesquiterpene lactone helenalin inhibits the transcription factor NF-kappaB by directly targeting p65. J Biol Chem 273: 33508-33516, 1998.

20. Rasul A, Bao R, Malhi M, Zhao B, Tsuji I, Li J and Li X Induction of apoptosis by costunolide in bladder cancer cells is mediated through ROS generation and mitochondrial dysfunction. Molecules 18: 1418-1433, 2013.

21. Ferrari FC, Ferreira LC, Souza MR, Grabe-Guimarães A, Paula CA, Rezende SA and Saúde-Guimarães DA: Antiinflammatory sesquiterpene lactones from Lychnophora trichocarpha Spreng. (Brazilian Arnica). Phytother Res 27: 384-389, 2013.

22. Kretschmer N, Rinner B, Stuendl N, et al: Effect of costunolide and dehydrocostus lactone on cell cycle, apoptosis, and ABC transporter expression in human soft tissue sarcoma cells. Planta Med 78: 1749-1756, 2012

23. Piornedo Rdos R, de Souza P, Stefanello MÉ, Strapasson RL, Zampronio AR and Kassuya CA: Anti-inflammatory activity of extracts and 11,13-dihydrozaluzanin C from Gochnatia polymorpha ssp. floccosa trunk bark in mice. J Ethnopharmacol 133: 1077-1084, 2011.

24. Collins SJ, Ruscetti FW, Gallagher RE and Gallo RC: Terminal differentiation of human promyelocytic leukemia cells induced by dimethyl sulfoxide and other polar compounds. Proc Natl Acad Sci USA 75: 2458-2462, 1978 .

25. Tanaka H, Abe E, Miyaura C, Shiina $Y$ and Suda $T$ : 1 alpha,25-dihydroxyvitamin D3 induces differentiation of human promyelocytic leukemia cells (HL-60) into monocytemacrophages, but not into granulocytes. Biochem Biophys Res Commun 117: 86-92, 1983 .

26. Kim SH, Danilenko M and Kim TS: Differential enhancement of leukaemia cell differentiation without elevation of intracellular calcium by plant-derived sesquiterpene lactone compounds. Br J Pharmacol 155: 814-825, 2008.
27. Song JH, Kim HJ, Lee CH, Kim SJ, Hwang SY and Kim TS: Identification of gene expression signatures for molecular classification in human leukemia cells. Int J Oncol 29: 57-64, 2006.

28. Pan Y, Chen H, Siu F and Kilberg MS: Amino acid deprivation and endoplasmic reticulum stress induce expression of multiple activating transcription factor-3 mRNA species that, when overexpressed in HepG2 cells, modulate transcription by the human asparagine synthetase promoter. J Biol Chem 278: 38402-38412, 2003.

29. Rousseau J, Gagné V, Labuda M, et al: ATF5 polymorphisms influence ATF function and response to treatment in children with childhood acute lymphoblastic leukemia. Blood 118 : 5883-5890, 2011.

30. Kang SN, Kim SH, Chung SW, Lee MH, Kim HJ and Kim TS Enhancement of 1 alpha,25-dihydroxyvitamin D(3)-induced differentiation of human leukaemia HL-60 cells into monocytes by parthenolide via inhibition of NF-kappa B activity. Br J Pharmacol 135: 1235-1244, 2002.

31. Gutierrez JA, Pan YX, Koroniak L, Hiratake J, Kilberg MS and Richards NG: An inhibitor of human asparagine synthetase suppresses proliferation of an L-asparaginase-resistant leukemia cell line. Chem Biol 13: 1339-1347, 2006.

32. Lorenzi PL, Reinhold WC, Rudelius M, et al: Asparagine synthetase as a causal, predictive biomarker for L-asparaginase activity in ovarian cancer cells. Mol Cancer Ther 5: 2613-2623, 2006.

33. Clavell LA, Gelber RD, Cohen HJ, et al: Four-agent induction and intensive asparaginase therapy for treatment of childhood acute lymphoblastic leukemia. N Engl J Med 315: 657-663, 1986.

34. Ortega JA, Nesbit ME Jr, Donaldson MH, Hittle RE, Weiner J, Karon $\mathrm{M}$ and Hammond D: L-Asparaginase, vincristine, and prednisone for induction of first remission in acute lymphocytic leukemia. Cancer Res 37: 535-540, 1977.

35. Hongo S, Sakagami H and Sato T: Decrease in asparagine synthetase activity during cell differentiation of mouse and human leukemia cell lines. Leukemia 4: 708-711, 1990.

36. Hashimoto K, Suzuki F and Sakagami H: Declined asparagine synthetase mRNA expression and enhanced sensitivity to asparaginase in HL-60 cells committed to monocytic differentiation. Anticancer Res 29: 1303-1308, 2009. 